\title{
IGR J17544-2619: a new supergiant fast X-ray transient revealed by optical/infrared observations ${ }^{\star}$
}

\author{
L. J. Pellizza ${ }^{1}$, S. Chaty ${ }^{1}$, and I. Negueruela ${ }^{2}$ \\ 1 AIM - Astrophysique Interactions Multi-échelles (Unité Mixte de Recherche 7158 CEA/CNRS/Université Paris 7 Denis Diderot), \\ CEA Saclay, DSM/DAPNIA/Service d'Astrophysique, Bât. 709, L'Orme des Merisiers, 91191 Gif-sur-Yvette Cedex, France \\ e-mail: lpellizz@discovery.saclay.cea.fr \\ 2 Dpto. de Física, Ingeniería de Sistemas y Teoría de la Señal, Universidad de Alicante, Apdo. 99, E03080 Alicante, Spain
}

Received 28 October 2005 / Accepted 10 May 2006

\begin{abstract}
One of the most recent discoveries of the INTEGRAL observatory is the existence of a previously unknown population of X-ray sources in the inner arms of the Galaxy. IGR J17544-2619, IGR J16465-4507 and XTE J1739-302 are among these sources. Although the nature of these systems is still unexplained, the investigations of the optical/NIR counterparts of the two last sources, combined with high energy data, have provided evidence of them being highly absorbed high mass X-ray binaries with blue supergiant secondaries and displaying fast X-ray transient behaviour. In this work we present our optical/NIR observations of IGR J17544-2619, aimed at identifying and characterizing its counterpart. We show that the source is a high mass X-ray binary at a distance of 2-4 kpc with a strongly absorbed O9Ib secondary, and discuss the nature of the system.
\end{abstract}

Key words. X-rays: binaries - X-rays: individual: IGR J17544-2619

\section{Introduction}

In recent years, a set of new X-ray sources were discovered by INTEGRAL and RXTE observatories within a few tens of degrees of the direction to the galactic center (Negueruela 2004; Kuulkers 2005). These sources present hard X-ray spectra, usually interpreted as due to strong absoption by large hydrogen column densities arising from circumstellar material. Their continuum spectral parameters are typical of neutron stars or black holes. They are suspected to be high mass X-ray binaries $(\mathrm{HMXBs})$ embedded in highly absorbing media, and for some of them massive companions were identified (e.g., Filliatre \& Chaty 2004; Smith 2004; Masetti et al. 2006; Negueruela et al. 2005, 2006). Among these sources there are transient ones, characterized by very short outbursts ( hours) separated by large quiescence periods (Smith et al. 2006; in't Zand 2005). IGR J16465-4507 and XTE J1739-302/IGR J17391-3021 are two of these transient sources, which have been shown to be HMXBs with highly reddened early type supergiant secondaries (Smith 2004; Negueruela et al. 2005, 2006), hence supporting the hypothesis of these sources being surrounded by large amounts of material.

IGR J17544-2619 is a fast transient source lying in the direction of the galactic center $\left(l=3.24^{\circ}, b=-0.34^{\circ}\right)$. It was discovered by the IBIS/ISGRI instruments onboard INTEGRAL on September 17, 2003 (Sunyaev et al. 2003), during an outburst which lasted around $2 \mathrm{~h}$. A second outburst lasting for $8 \mathrm{~h}$ was observed the same day (Grebenev et al. 2003), and a third one lasting for $10 \mathrm{~h}$ was detected on March 8, 2004 (Grebenev et al. 2004), demonstrating that this is a recurrent

* Based on observations collected at the European Southern Observatory, Chile, under proposal ESO 71.D-0073. transient source. An oscillation-like behaviour with a time scale of 1.5-2 $\mathrm{h}$ has also been detected in the March 2004 observation (Grebenev et al. 2004). The source has also been observed by XMM-Newton on March 17 and September 11 and 17, 2003 (González-Riestra et al. 2004) and by Chandra on July 3, 2004 (in't Zand 2005). XMM-Newton observations revealed a hard spectrum, that can be modelled by an absorbed power law with either variable absorption or variable spectral index, in both cases with a large hydrogen column density $\left(N_{\mathrm{H}} \sim\right.$ $1.9-4.3 \times 10^{22} \mathrm{~cm}^{-2}$ ). Chandra observations also showed a hard spectrum during bursts, but a softer one in quiescence, with similar absorption $\left(N_{\mathrm{H}}=1.36 \pm 0.22 \times 10^{22} \mathrm{~cm}^{-2}\right)$. Assuming a distance of $8 \mathrm{kpc}$, total unabsorbed luminosities of the source in the $0.5-10 \mathrm{keV}$ range are of the order of $10^{32} \mathrm{erg} \mathrm{s}^{-1}$ in quiescence and $10^{35}-10^{36} \mathrm{erg} \mathrm{s}^{-1}$ during activity. These values are typical of HMXBs containing either neutron stars or black holes. XMM-Newton and Chandra observations also allowed a precise positioning of the source $\left(4^{\prime \prime}\right.$ and $0.6^{\prime \prime}$ error boxes respectively), challenging its identification with the ROSAT source 1RXS J175428.3-2620 proposed by Wijnands (2004), and supporting the optical/NIR counterpart candidate (USNOB1.0 0636-0620933/2MASS J17542527-2619526) suggested by Rodriguez (2003). The XMM-Newton ultraviolet magnitudes, combined with optical/NIR data are also consistent with IGR J17544-2619 having an early O-type companion (González-Riestra et al. 2004).

One day after the discovery of IGR J17544-2619, we triggered our ESO Target of Opportunity program to obtain optical and NIR images of the field of this source, and optical spectra of the counterpart candidate proposed by Rodriguez (2003), which is also the brightest object in the XMM-Newton error box. These measurements were aimed at confirming the optical/NIR 
Table 1. Optical and NIR imaging log.

\begin{tabular}{cccccc}
\hline \hline Filter & $\begin{array}{c}\text { Exposure } \\
\text { time }(\mathrm{s})\end{array}$ & \# frames & Filter & $\begin{array}{c}\text { Exposure } \\
\text { time }(\mathrm{s})\end{array}$ & \# frames \\
\hline$B$ & 300 & 1 & $J$ & 60 & 9 \\
$V$ & 200 & 1 & $H$ & 60 & 9 \\
$V$ & 10 & 90 & $K_{\mathrm{s}}$ & 60 & 9 \\
$R$ & 200 & 1 & $K_{\mathrm{s}}$ & 2 & 366 \\
\hline
\end{tabular}

Table 2. Spectroscopy log.

\begin{tabular}{lccc}
\hline \hline $\begin{array}{l}\text { Grating } \\
\text { or grism }\end{array}$ & $\begin{array}{c}\text { Exposure } \\
\text { time }(\mathrm{s})\end{array}$ & $\begin{array}{c}\text { Wavelength } \\
\text { range }(\AA)\end{array}$ & $\begin{array}{c}\text { Resolution } \\
(\AA)\end{array}$ \\
\hline$\# 3$ & 150 & $3600-8400$ & 7.9 \\
$\# 5$ & 400 & $3800-6700$ & 4.5 \\
$\# 6$ & 300 & $6180-6860$ & 1.2 \\
$\# 12$ & 700 & $4000-4920$ & 2.6 \\
\hline
\end{tabular}

counterpart and determining its properties. In this paper we present our observations (Sect. 2), their results (Sect. 3), and discuss the nature of the system (Sect. 4).

\section{Observations}

Our observations of IGR J17544-2619 were carried out on the nights of September 18, 2003 (imaging) and May 11, 2004 (spectroscopy), with the ESO 3.5-meter New Technology Telescope (NTT) at La Silla Observatory, Chile. Optical and NIR images of the field of the source were obtained with the ESO Multi-Mode Instrument (EMMI) and the Son Of Isaac (SOFI) instruments respectively, as part of a Target of Opportunity program (ESO 71.D-0073, P. I. Chaty). Intermediate resolution optical spectra of the counterpart candidate proposed by Rodriguez (2003) were also obtained with EMMI.

The red arm of EMMI, equipped with a mosaic of two $2048 \times 4096$ MIT CCD detectors, was used in the longslit REMD mode together with grating \#6 and in RILD mode with grisms \#3 and \#5 for spectroscopy. The same arm of EMMI was used in RILD mode with $B, V$ and $R$ Bessel filters for imaging. The blue arm, equipped with a Textronik TK1034 thinned, backilluminated $1024 \times 1024 \mathrm{CCD}$, was also used for spectroscopy in the longslit BLMD mode with grating \#12. SOFI, equipped with a Rockwell Hg:Cd:Te $1024 \times 1024$ Hawaii array, was used with the Large Field Objective and $J, H$ and $K_{\mathrm{s}}$ filters for NIR imaging.

We took a set of very deep images in each filter in order to search for possible counterpart candidates not detected by previous surveys, and to perform accurate photometry of all candidates. Due to the nature of the high energy source, photometric variability is expected, hence two sets of short, contiguous exposures spanning $1-2 \mathrm{~h}$ were also taken to construct optical and NIR light curves respectively. We took also long exposure spectra of USNO-B1.0 0636-0620933 in order to cover the whole optical spectral range with a high $\mathrm{S} / \mathrm{N}$ ratio. Tables 1 and 2 give the basic parameters used for the observations. The standard reduction procedures for optical and NIR images (sky/bias substraction and flat-fielding) were used to obtain the final science images. The reduction was performed using the IRAF package CCDRED (Tody 1993). The spectra were pre-processed with MIDAS and then reduced using the Starlink packages CCDPACK and FIGARO.

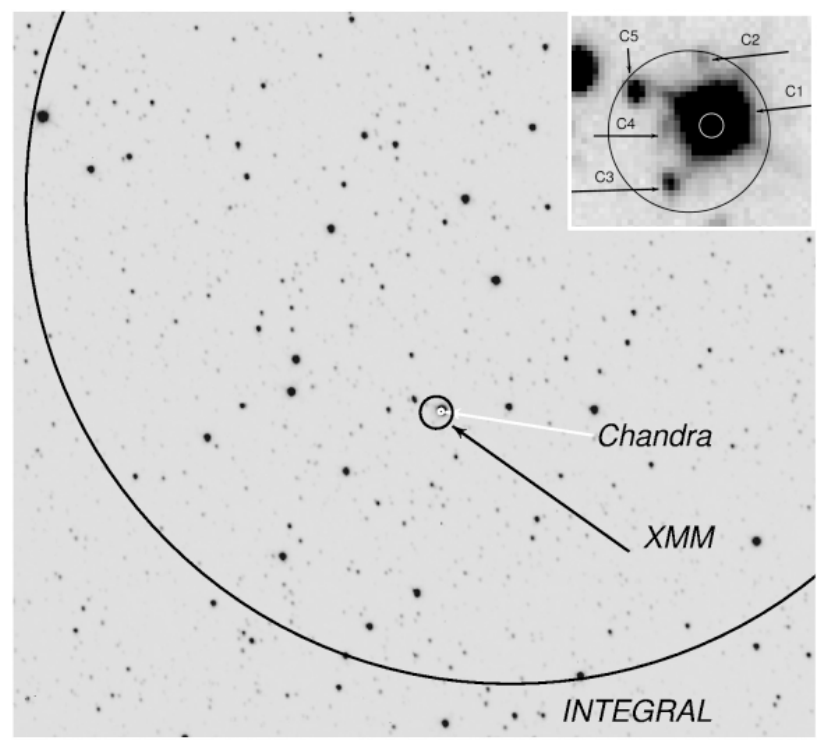

Fig. 1. $K_{\mathrm{s}}$ band image of the field of IGR J17544-2619. North is up and East is to the left. The large circular section is the INTEGRAL error circle ( $2^{\prime}$ radius) for the source, while the small circles are the XMM-Newton and Chandra error boxes (black, 4" and white, 0.6" radius respectively). Inset: the five counterpart candidates (C1-C5) found inside the XMM-Newton error box (black circle). The brightest candidate $(\mathrm{C} 1)$, the only one allowed by the Chandra error box (white circle), is USNO-B1.0 0636-0620933/2MASS J17542527-2619526, the candidate proposed by Rodriguez (2003). The image was processed to unveil the faintest candidates, hence the intensity scale is nonlinear.

\section{Data analysis and results}

\subsection{Astrometry}

We performed the astrometry of our images to determine the position in our frames of the XMM-Newton and Chandra error boxes for IGR J17544-2619 and to search for optical/NIR counterparts within them. We chose the best frame in each band for this purpose, and selected from it a set of bright pointlike objects in uncrowded regions. We took the coordinates of these objects from the USNO B1.0 and the 2MASS catalogs (Monet et al. 2003; Cutri et al. 2003), and computed the plate solution for each frame using the IRAF CCMAP task, obtaining an rms error $<0.15^{\prime \prime}$, which is small enough for our purposes. In Fig. 1 we show the field of the source in the $K_{\mathrm{s}}$ band, together with the INTEGRAL, XMM-Newton and Chandra error circles. Inside the second one we found 5 counterpart candidates $(\mathrm{C} 1-\mathrm{C} 5$, their positions are given in Table 3). The brightest candidate (C1) is the one proposed by Rodriguez (2003), the other four (including one apparently extended object, C4) are only visible in the NIR and are extremely faint, close to our detection limit. The Chandra error box clearly excludes all the candidates but $\mathrm{C} 1$, hence we conclude that this is the correct counterpart of IGR J17544-2619. The three faint pointlike objects are probably foreground dwarf stars, while the extended one might be a background galaxy.

\subsection{Spectroscopy}

Spectroscopic observations of candidate $\mathrm{C} 1$ enabled us to determine for the first time its spectral type. In Fig. 2 we present the optical spectra of this object taken with grisms \#12 and \#3, while in Table 4 we list the spectral features identified in it. The presence of intense He II lines in the spectrum of $\mathrm{C} 1$ suggests an $\mathrm{O}$ spectral type. According to the 


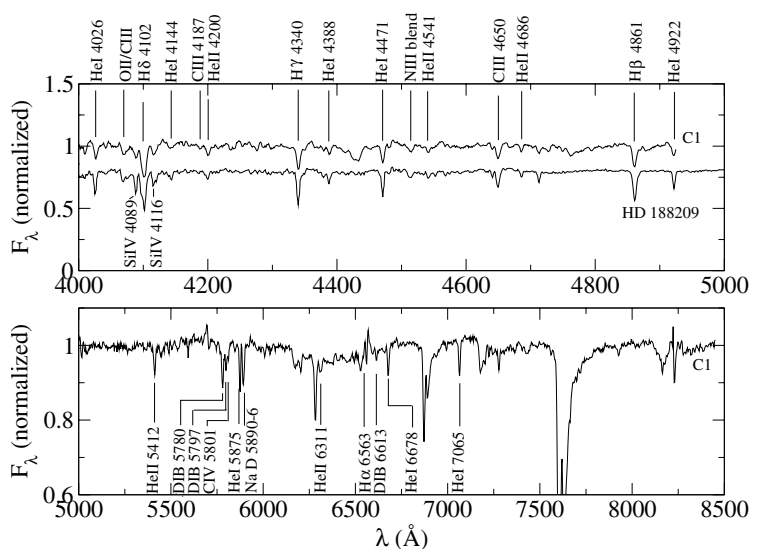

Fig. 2. Optical spectrum of USNO-B1.0 0636-0620933 showing the identified lines, among which we found strong He II lines typical of an O-type star. Upper panel: blue spectrum of $\mathrm{C} 1$ taken with grism \#12 (upper curve), and the standard O9Ib star HD 188209 (lower curve). The high degree of similarity between them supports our classification of $\mathrm{C} 1$ as an O9Ib star. Lower panel: red spectrum of C1 between $5000 \AA$ and $8500 \AA$, taken with grism \#3. The features at $6850 \AA$ and $7600 \AA$ are of telluric origin.

Table 3. Positions of optical/NIR counterpart candidates of the high energy source IGR J17544-2619, and their distances to the brightest one.

\begin{tabular}{ccccl}
\hline \hline Id. & $\begin{array}{c}\alpha(\mathrm{J} 2000) \\
(\mathrm{h} \mathrm{m} \mathrm{s})\end{array}$ & $\begin{array}{c}\delta(\mathrm{J} 2000) \\
\left({ }^{\circ}{ }^{\prime \prime}\right)\end{array}$ & $\begin{array}{c}\text { Distance } \\
\text { to C1 }\left({ }^{\prime \prime}\right)\end{array}$ & Notes \\
\hline C1 & $17: 54: 25.27$ & $-26: 19: 52.7$ & 0.0 & Bright \\
C2 & $17: 54: 25.32$ & $-26: 19: 49.7$ & 3.1 & Faint, pointlike \\
C3 & $17: 54: 25.44$ & $-26: 19: 55.8$ & 3.9 & Faint, pointlike \\
C4 & $17: 54: 25.46$ & $-26: 19: 53.2$ & 2.6 & Faint, extended? \\
C5 & $17: 54: 25.57$ & $-26: 19: 51.2$ & 4.3 & Faint, pointlike \\
\hline
\end{tabular}

classification criteria of Walborn \& Fitzpatrick (1990), the ratio He II $4541 \AA / \mathrm{He}$ I $4471 \AA$ indicates that $\mathrm{C} 1$ is an O9 star. The luminosity class of $\mathrm{C} 1$ could also be determined using the intensity of the He II $4686 \AA$ as a luminosity indicator (Walborn $\&$ Fitzpatrick 1990). This line is very weak, but clearly visible in absorption in our object, indicating a Ib luminosity class. Due to the low $\mathrm{S} / \mathrm{N}$ ratio of the spectrum, there is some uncertainty about this spectral classification, but the O9Ib spectral type is fully supported by the lower resolution, higher $\mathrm{S} / \mathrm{N}$ ratio grism \#5 spectrum. Figure 2 includes, for comparison, the spectrum of the O9Ib star HD 188209, showing that the general appearance of both spectra is similar. This classification confirms that $\mathrm{C} 1$ is a blue supergiant and, via the calibration of Drilling \& Landolt (1999), provides the first determination of its mass, 25-28 $M_{\odot}$. Hence, we conclude that IGR J17544-2619 is a HMXB. Another interesting feature of the $\mathrm{C} 1$ spectrum is the $\mathrm{H} \alpha$ line, that shows a P-Cygni type profile. This is the signature of mass loss through a strong stellar wind, present in normal O-type stars.

The spectrum of $\mathrm{C} 1$ shows also several diffuse interstellar bands (DIBs), usually present in the spectra of sources with large extinction. The DIBs at $5780 \AA, 5797 \AA$ and $6613 \AA$ correlate with the color excess $E(B-V)$ (e.g., Herbig 1993; Cox et al. 2005), hence we can use them to determine the latter. In our spectra, the DIB at $5797 \AA$ is blended with the C IV $5801 \AA$ line, but the other two DIBs are clearly resolved. The first one shows an equivalent width $W_{5780}=1020 \pm 80 \mathrm{~m} \AA$ implying, through the relationship given by Herbig (1993), a color excess
Table 4. Absorption lines identified in the spectrum of USNOB1.0 0636-0620933, and the atomic species that produce them.

\begin{tabular}{cclccl}
\hline \hline $\begin{array}{c}\lambda_{\text {obs }} \\
(\AA)\end{array}$ & $\begin{array}{c}\lambda_{\text {lab }} \\
(\AA)\end{array}$ & Species & $\begin{array}{c}\lambda_{\text {obs }} \\
(\AA)\end{array}$ & $\begin{array}{c}\lambda_{\text {lab }} \\
(\AA)\end{array}$ & Species \\
\hline $4027 \pm 1$ & 4026.189 & He I & $4542 \pm 1$ & 4541.590 & He II \\
& 4026.362 & He I & $4650 \pm 1$ & 4650.160 & C III \\
$4070 \pm 1$ & 4067.870 & C III & & 4651.350 & C III \\
& 4068.970 & C III & $4685 \pm 1$ & 4685.682 & He II \\
& 4069.636 & O II & $4861 \pm 1$ & 4861.332 & H $\beta$ \\
& 4069.897 & O II & $4921 \pm 1$ & 4921.929 & He I \\
& 4070.300 & C III & $5412 \pm 3$ & 5411.524 & He II \\
$4089 \pm 1$ & 4088.863 & Si IV & $5881 \pm 3$ & 5875.618 & He I \\
$4101 \pm 1$ & 4101.737 & H $\delta$ & & 5875.650 & He I \\
$4115 \pm 1$ & 4116.104 & Si IV & & 5875.989 & He I \\
$4142 \pm 1$ & 4143.759 & He I & & 5889.953 & Na I \\
$4187 \pm 1$ & 4187.050 & C III & & 5895.923 & Na I \\
$4200 \pm 1$ & 4199.830 & He II & $6310 \pm 3$ & 6310.800 & He II \\
$4340 \pm 1$ & 4340.468 & H $\gamma$ & $6564 \pm 1$ & 6562.817 & H $\alpha$ \\
$4388 \pm 1$ & 4387.928 & He I & $6679 \pm 3$ & 6678.149 & He I \\
$4471 \pm 1$ & 4471.477 & He I & $7062 \pm 3$ & 7065.188 & He I \\
& 4471.688 & He I & & 7065.719 & He I \\
$4515 \pm 1$ & 4510.920 & N III & & & \\
& 4514.890 & N III & & & \\
\hline
\end{tabular}

$E(B-V)=1.97 \pm 0.15$. For the other one, our measured equivalent width $\left(W_{6613}=300 \pm 50 \mathrm{~m} \AA\right)$ is contained in the region where the relationship between $W$ and $E(B-V)$ is nonlinear. Figure 4 of Cox et al. (2005) shows that this value is consistent with $E(B-V)>1$, the flattening of the relationship preventing any other meaningful conclusion.

\subsection{Photometry}

Differential aperture photometry of $\mathrm{C} 1$ was made using the IRAF package APPHOT. For each frame, we measured its instrumental optical and NIR magnitudes $m_{B}, m_{V}, m_{R}, m_{J}, m_{H}$ and $m_{K_{\mathrm{s}}}$, together with those of 18 comparison stars with known USNO B1.0 and 2MASS magnitudes. The Johnson $B, V$ and $R$ magnitudes of the comparison stars were computed from their photographic IIIa-J (blue) and IIIa-F (red) magnitudes taken from the USNO B1.0 catalog and the transformations of Windhorst et al. (1991), while their $J, H$ and $K_{\mathrm{s}}$ standard magnitudes were taken directly from the $2 \mathrm{MASS}$ catalog.

Linear fits of the standard optical magnitudes against corresponding instrumental magnitudes present slopes consistent with unity within errors of a few percent, showing that our instrumental magnitudes differ from the standard system only in the zero points for the different bands. These were computed by taking the mean of the differences $B-m_{B}, V-m_{V}$ and $R-m_{R}$ for the comparison stars. Their uncertainties were determined by assuming that these differences follow a normal distribution, and computing the error of the mean. The standard deviation of these differences is $\sim 0.2 \mathrm{mag}$, consistent with the facts that USNO B1.0 uncertainties are around $0.3 \mathrm{mag}$ in the worst cases (Monet et al. 2003) and that the transformations between photographic and Johnson systems add an error of $\sim 0.1 \mathrm{mag}$ in quadrature (Windhorst et al. 1991). Hence, our zero points have uncertainties of $\sim 0.05 \mathrm{mag}$.

For NIR images, C1 was slightly in the nonlinear range of SOFI CCDs, hence quadratic fits of standard 2MASS magnitudes $J, H$ and $K_{\mathrm{s}}$ against corresponding instrumental magnitudes $m_{J}, m_{H}$ and $m_{K_{\mathrm{s}}}$ respectively were used to derive the NIR magnitudes of $\mathrm{C} 1$. The errors of the estimated magnitudes were 
Table 5. Optical and NIR magnitudes of the counterpart candidates of the high energy source IGR J17544-2619. The lower limits stand for the combined magnitudes of the four faint candidates C2-C5.

\begin{tabular}{lcccccc}
\hline \hline Id. & $B$ & $V$ & $R$ & $J$ & $H$ & $K_{\mathrm{s}}$ \\
& $\begin{array}{c} \pm 0.05 \\
(\mathrm{mag})\end{array}$ & $\begin{array}{c} \pm 0.05 \\
(\mathrm{mag})\end{array}$ & $\begin{array}{c} \pm 0.02 \\
(\mathrm{mag})\end{array}$ & $\begin{array}{c} \pm 0.02 \\
(\mathrm{mag})\end{array}$ & $\begin{array}{c} \pm 0.02 \\
(\mathrm{mag})\end{array}$ & $\begin{array}{c}\mathrm{mag}) \\
\end{array}$ \\
\hline C1 & 14.44 & 12.65 & $<11.9$ & 8.71 & 8.03 & 7.99 \\
C2-5 & $>19.5$ & $>17.7$ & $>16.2$ & $>13.8$ & $>13.1$ & $>13.1$ \\
\hline
\end{tabular}

computed from the full covariance matrix of the fitted parameters, and take into account the uncertainties of the 2MASS magnitudes of the comparison stars, our instrumental magnitude errors being much smaller.

The optical and NIR magnitudes of $\mathrm{C} 1$ are shown in Table 5. The value of $R$ is an upper limit as the image of C1 was slightly saturated in this band. We performed the photometry for different aperture diameters $d$ from $1^{\prime \prime}$ to $5^{\prime \prime}$ in order to detect any flux contribution from the fainter candidates. In all cases, the constancy of the $\mathrm{C} 1$ instrumental magnitudes for $d>2.5^{\prime \prime}$ up to an accuracy of $0.01 \mathrm{mag}$ allows us to put conservative lower limits for the combined magnitude of all of them (see Table 5). The contribution of $\mathrm{C} 2-\mathrm{C} 5$ is thus negligible and we can ensure that it does not contaminate our results on $\mathrm{C} 1$ at the $0.01 \mathrm{mag}$ accuracy level.

Our broad-band magnitudes are in accordance with those listed in Rodriguez (2003), and also with the $B$ magnitude measured by XMM-Newton (González Riestra et al. 2004). We note that our $J$ and $H$ magnitudes differ (the first one marginally) from those given by $2 \mathrm{MASS}$, our estimates being lower than catalog values; but our $K_{\mathrm{s}}$ value agrees well with $2 \mathrm{MASS}$ data $\left(J_{\text {2MASS }}=8.791 \pm 0.021, H_{2 \text { MASS }}=8.310 \pm 0.031, K_{\mathrm{s}, 2 \mathrm{MASS}}=\right.$ $8.018 \pm 0.026)$. Because of the high accuracy of both 2 MASS and our measurements, and the fact that our determinations are based on the 2MASS magnitudes of the comparison stars, we conclude that the differences might arise in a true variation in the NIR flux of C1, brighter in September 2003 than in the epoch of 2MASS observations (July 1998). This variation makes a stronger case for the identification of $\mathrm{C} 1$ as the correct counterpart of IGR J17544-2619. We suggest that it might be related to the X-ray activity of the source. A more detailed study through simultaneous multiwavelength campaigns both during quiescence and activity would be important to confirm these points.

The combination of the spectral type of $\mathrm{C} 1$ with its photometry gave us a deeper insight into the nature of the system. An O9Ib star has an intrinsic colour $(B-V)_{0}=-0.28 \pm 0.01$ (Schmidt-Kaler 1982), whereas we measure $B-V=1.79 \pm 0.10$, implying a reddening $E(B-V)=2.07 \pm 0.11$. This value is consistent with that found using the DIBs observed in the spectra of $\mathrm{C} 1$. Hereafter we use the average of both, $E(B-V)=$ $2.02 \pm 0.13$. Assuming a standard extinction law $\left(R_{V}=3.1\right)$ gives a total visual extinction $A_{\mathrm{V}}=6.26 \pm 0.40$. There is no reliable absolute visual magnitude calibration for O9Ib stars alone, but as Ib stars are the least luminous objects of class I, we take the mean value for all O9I stars, $M_{V}=-6.29$ (Martins et al. 2005), as an upper limit for the luminosity of $\mathrm{C} 1$. On the other hand, the faintest O9I stars have $M_{V} \sim-5.6$ (Martins et al. 2005). With this interval for the absolute visual magnitude of $\mathrm{C} 1$, we derive a range of $2.1-4.2 \mathrm{kpc}$ for the distance of the system, far smaller than the $8.5 \mathrm{kpc}$ computed by Smith (2004) using USNO B1.0 photometry. The disagreement arises from the lack of accuracy of USNO magnitudes. This result is very robust,



Fig. 3. Spectral energy distribution $F_{\lambda}(\lambda)$ of the source. Squares represent the fluxes observed by us and triangles those from XMM-Newton and 2MASS data. Circles correspond to extinction corrected fluxes. The solid line shows the SED of a blackbody at the effective temperature of an O9Ib star $\left(T_{\text {eff }}=3.1 \times 10^{4} \mathrm{~K}\right)$, normalized to have the same unabsorbed $V$ magnitude of the source.

even if $\mathrm{C} 1$ were among the brightest O9I stars, the distance would be still smaller than $\sim 5 \mathrm{kpc}$, and if a value $R_{V}>3.1$ were used, the distance would decrease. Hence, the most probable localization of IGR J17544-2619 is between 2-4 kpc of the Sun, in the Scutum-Crux arm of the Milky Way (see Vallée 2005 for a model of the Milky Way spiral arms). We note that small distances were also obtained for XTE J1739-302 (1.8-2.9 kpc; Negueruela et al. 2006) and IGR J16318-4848 (<4 kpc; Filliatre \& Chaty 2004).

The observed extinction implies a hydrogen column density $N_{\mathrm{H}}=1.2 \times 10^{22} \mathrm{~cm}^{-2}$, close to the interstellar value $(1.4 \times$ $10^{22} \mathrm{~cm}^{-2}$, Dickey \& Lockman 1990). Our value is consistent with those obtained from Chandra observations $(1.36 \pm 0.22 \times$ $\left.10^{22} \mathrm{~cm}^{-2}\right)$ and lower than XMM-Newton results $(1.9-4.3 \times$ $10^{22} \mathrm{~cm}^{-2}$ ), showing that the higher $N_{\mathrm{H}}$ values are obtained during X-ray activity, $N_{\mathrm{H}}$ decreasing during quiescence. This suggests the existence of a highly variable circumstellar medium around the system, whose presence is related to the high energy activity of the source. We note that the possibility of the extinction being always completely interstellar is difficult to reconcile with the variable $N_{\mathrm{H}}$ suggested by the combination of X-ray and our observations.

The photometry obtained allowed us to construct for the first time a spectral energy distribution (SED) of the source, which we show in Fig. 3. Squares in this figure represent our measurements of the observed flux of the source $F_{\lambda}$ as a function of wavelength $\lambda$, while triangles represent XMM-Newton and 2MASS data. Using the estimated visual extinction and its relationship to the extinction in other bands (Mathis 1999), we computed the latter and corrected the SED for its effects (circles in Fig. 3). We also constructed the SED of an O9Ib star normalized to have the same unabsorbed $V$ magnitude of $\mathrm{C} 1$, using its intrinsic color indices (Drilling \& Landolt 1999; Tokunaga 1999). We found that it is very well fitted by a blackbody with a temperature equal to the effective temperature of an O9I star $\left(T_{\text {eff }}=3.1 \times 10^{4} \mathrm{~K}\right.$, Martins et al. 2005), normalized in the same way (solid line in Fig. 3). We note the very good agreement between the two unabsorbed SEDs in the optical, NIR and part of the UV, confirming our assumption of standard reddening. The lack of detection of $\mathrm{C} 1$ in the $U V M 2$ band $(\lambda=0.234 \mu \mathrm{m}$, 


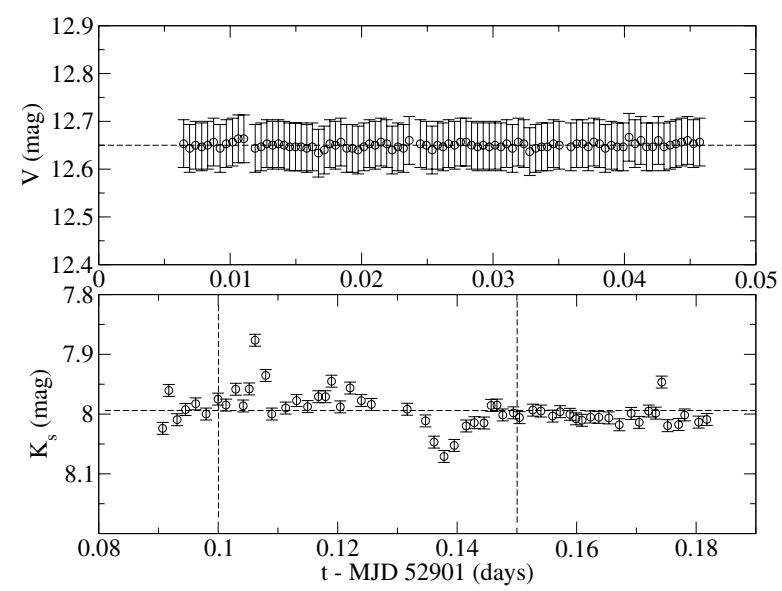

Fig. 4. Optical and NIR light curves of $\mathrm{C} 1$. Abscissas are given as Modified Julian Dates (MJD), MJD 52901 being September 19, 2003, $0^{\mathrm{h}}$ UT. Upper panel: the $V$ band light curve is extremely flat, showing a constant contribution from the $\mathrm{O}$ star. Lower panel: the $K_{\mathrm{s}}$ band light curve shows erratic variations around $t$-MJD $52901 \sim 0.10-0.15$ days (between the two dashed lines), note the two brightenings ( $\sim 0.10 \mathrm{mag}$ at 0.105 days and $\sim 0.05 \mathrm{mag}$ at 0.12 days $)$ and the fading ( $\sim 0.10 \mathrm{mag}$ at 0.14 days). The differences with the $V$ band curve suggest that they are probing different parts of the system, and supports the picture of the existence of circumstellar material around the star.

González-Riestra et al. 2004) is consistent with the blackbody spectrum and the amount of extinction derived by us. However, the detection at $0.218 \mu \mathrm{m}$ ( $U V W 2$ band) is puzzling, because the absorbed flux should be more than two magnitudes below the limiting magnitude of the XMM-Newton OM for this wavelength (there is a strong enhancement in the extinction curve at this wavelength, the $2175 \AA$ bump). A peculiar value for the extinction at $0.218 \mu \mathrm{m}$ (e.g., due to the depletion of the material that produces the $2175 \AA$ bump) could be one solution, but it is improbable given the good agreement in other wavelengths and the fact that a reduction of $\sim 9$ mag in the extinction in the $U V W 2$ band is needed to restore the agreement with the blackbody spectrum. On the other hand, if the computed absorption is correct, the measured magnitude $\left(m_{U V W 2}=14.49 \pm 0.02\right) \mathrm{im}$ plies that the unabsorbed flux at this wavelength is more than three orders of magnitude greater than the blackbody estimate. This disagreement could be explained if the source were variable in the $\mathrm{UV}$, because the observations in the $U V W 2$ and $U V W 1$ $(\lambda=0.295 \mu \mathrm{m})$ bands were separated by 6 days. If this is the case, it would be interesting also to determine if the variability is connected to the X-ray behaviour. Simultaneous multiwavelength campaigns are clearly needed to investigate this issue and determine the astrophysical processes which operate in this source.

\subsection{Light curves}

Using the $90 \mathrm{~V}$-band and the $366 K_{\mathrm{s}}$-band short exposures (see Table 1), we constructed optical/NIR light curves of C1 spanning $3.4 \mathrm{ks}$ and $8 \mathrm{ks}$ respectively (Fig. 4). The NIR data were averaged in groups of 4 , which results in a lower uncertainty of $\sim 0.01 \mathrm{mag}$. The $V$ light curve is flat and completely featureless down to our accuracy level of $0.05 \mathrm{mag}$. The $K_{\mathrm{s}}$ light curve, instead, shows an erratic behaviour barely above our accuracy limits of $0.01 \mathrm{mag}$. A few sporadic events are seen around $t$ - MJD $52901 \sim 0.10-0.15$ days, including two short brightenings of approximately $0.05-0.10 \mathrm{mag}$ and an abrupt fading of about $0.10 \mathrm{mag}$, reminiscent of an eclipse. No periodicity is apparent in our light curves.

Although the optical and NIR light curves are not simultaneous, they are close in time, hence their different behaviours suggest that they are probing different parts of the system. The extreme flatness of the optical light curve contrasts with the multiple short erratic variations found in the NIR light curve. The $V$ light curve is probably reflecting the behaviour of the O9Ib star, as it is the brightest source in this spectral domain. We note that the constant light curve observed is not in contradiction with the fact that most $\mathrm{O}$ stars are microvariable in $V$ (e.g., Balona 1992), because their amplitudes are $<0.1 \mathrm{mag}$, lower than or of the same magnitude of our accuracy $( \pm 0.05 \mathrm{mag})$. The NIR variations, that occur on timescales of the order of hours, could be generated by a different component. A possible candidate is a variable amount of warm material (either circumstellar or in an accretion disc) emitting in the NIR. We note that IGR J17544-2619 was recently detected in the medium IR $(8.59 \mu \mathrm{m}$ and $11.26 \mu \mathrm{m})$ by the VISIR instrument on VLT (Chaty et al., in prep.). We also note that the erratic variability observed in $K_{\mathrm{s}}$ could explain the differences between our NIR magnitudes and 2MASS values. Longer light curves in several optical and IR bands together with optical/NIR spectra would be needed to address this issue.

\section{Discussion}

Using optical and NIR imaging of the field of IGR J17544-2619 we identified the optical/NIR counterpart of this high energy source to be USNO-B1.0 06360620933/2MASS J17542527-2619526. We have obtained strong evidence for IGR J17544-2619 being a high mass $\mathrm{X}$-ray binary. Although our data do not give any clue about the accretor in the HMXB, they can be obtained from the X-ray data. The peak flux density scaled to $1 \mathrm{kpc}(0.5-1.9 \mathrm{Crab}$, for our distance range of $2.1-4.2 \mathrm{kpc}$ ) and the spectral index obtained by Chandra are consistent with those of black holes in the hard state. Applying the relationship of Gallo et al. (2003), we obtain an expected radio flux of $\sim 10-35 \mathrm{mJy}$ for this source if the accretor is a black hole. Hence, the lack of detection of IGR J17544-2619 in radio ( $3 \sigma$ upper limit of $7 \mathrm{mJy}$ at $0.61 \mathrm{GHz}$, Pandey et al. 2006) suggests that the compact object is rather a neutron star. This agrees with the results of in't Zand (2005), who shows that the X-ray spectrum of IGR J17544-2619 in quiescence can be satisfactorily fitted by a neutron star model with a luminosity of $\sim 5 \times 10^{32} \mathrm{erg} \mathrm{s}^{-1}$.

For the mass donor, our results show that it is an O9Ib blue supergiant with a mass of 25-28 $M_{\odot}$. Hence, at its birth the object should have been an $\mathrm{O}+\mathrm{O}$ binary, a rare system due to the high masses of its components and their short lifetimes (a $25 M_{\odot}$ star lives only $\sim 7 \mathrm{Myr}$ in the main sequence). This characteristic is consistent with the fact that we find IGR J17544-2619 in a star-forming region of the Galaxy (the Scutum-Crux arm at $\sim 3-4 \mathrm{kpc}$ ). It is not surprising then to find other systems with similar properties in nearby regions (Scutum-Crux and Norma arms). Although massive binaries undergo mass-transfer phases before the primary evolves into a compact object, recent results suggest that the maximum mass gained by the secondary could be as small as $10 \%$ of the mass transferred (e.g., Petrovic et al. 2005). This suggests that the mass of the primary should have been $\gtrsim 25 M_{\odot}$, and hence that such massive stars are not necessarily constrained to evolve into black holes.

Our data, combined with X-ray observations, suggest that this system is embedded in circumstellar material. We derive a 
moderately high hydrogen column density $N_{\mathrm{H}}=1.2 \times 10^{22} \mathrm{~cm}^{-2}$, that agrees well with Chandra results and is lower than that derived from XMM-Newton data (González-Riestra et al. 2004; in't Zand 2005). This confirms that the extinction of the system is variable, and hence it can not be completely interstellar. The existence of NIR variability with no optical correlation shown by our light curves could be explained if we assume that optical and NIR emission arise in different regions of the system. The optical radiation comes from the blue supergiant, hence at least part of the NIR should come from another region, the circumstellar environment being a good candidate. This scenario is interesting regarding the recent detection of IGR J17544-2619 in the medium IR.

Our analysis, together with other recents studies (Smith 2004; in't Zand 2005; Negueruela et al. 2006; Smith et al. 2006) suggest that the group of the sources discovered by INTEGRAL and RXTE observatories close to the direction of the galactic center is not homogeneous, it rather shows two distinct subgroups. The first one comprises HMXBs with persistent X-ray emission, large circumstellar absorption and supergiant mass donors (e.g., IGR J16318-4848, Filliatre \& Chaty 2004). The second group, dubbed supergiant fast $X$-ray transients (SFXT) by Negueruela et al. (2006) and Smith et al. (2006), includes also supergiant HMXBs, but with fast ( $\sim$ hours) transient X-ray emission, very low quiescence X-ray luminosity $\left(\leq 10^{34} \mathrm{erg} \mathrm{s}^{-1}\right)$, and moderate to high variable $\mathrm{X}$-ray extinction originated in the circumstellar environment. IGR J17544-2619 belongs to this second group, together with XTE J1739-302 (the prototype of the group, Negueruela et al. 2006), IGR J16465-4507, AX 1845.0-0433 and possibly XTE J1901+014. SFXTs include also at least three other sources, SAX J1818.6-1703, AX J1749.1-2733 (in't Zand 2005) and IGR J11215-5952 (Sidoli et al. 2006).

As pointed out by in't Zand (2005) and Negueruela et al. (2006), the physical mechanism driving the fast outbursts in SFXTs would be related neither to the compact object, as the group includes both neutron star and black hole HMXBs, nor to the low luminosity, as fast outbursts were observed also in Vela X-1 and Cygnus X-1. The accretion mechanism is then the most probable cause of the short duration of the outbursts. As supergiant HMXBs are believed to be powered by accretion from the strong wind of the secondary, the variation of the mass flux of the wind is probably the cause of the outbursts. The changing distance of the mass donor in an excentric orbit is an attractive posibility for explaining this mass flux variation, as it could also explain the low quiescent luminosity and small duty cycle of the X-ray source if the O star spends most of the orbital period far away from the accretor. We point out that this is consistent with the fact that recurrent outbursts have been recently found in an SFXT (IGR J11215-5952), with a period of 329 days (Sidoli et al. 2006). On the other hand, local inhomogeinities (clumps) in the wind were proposed as an alternative to explain the fast outbursts, as they are individually accreted by the compact object (in't Zand 2005). We note that, if as we propose, the variability of the circumstellar medium and the X-ray activity are correlated, then individual clump accretion could not explain the whole phenomenology. A global wind density variation (whether it is composed of clumps or not) would be a better explanation for the outbursts, provided that it lasts for a time of the order of a few hours.

Finally, we point out that a more detailed monitoring of this system would be important to completely unveil its properties and hence improve our knowledge on this kind of high energy sources. Particularly, simultaneous multiwavelength campaigns would be needed to precisely measure the SED both in quiescence and during activity, establish the existence of circumstellar material beyond any doubt, and determine its properties. The analysis of both long and short-term variability (including the search for a binary period and wind velocity variability) and their connection to high energy activity would also shed light over the physical mechanisms involved in the behaviour of these systems.

Acknowledgements. We would like to thank Dr. Marc Ribó for useful discussions and a careful reading of the manuscript, and the anonymous referee for suggestions which greatly improved this paper. IN is a researcher of the program Ramón y Cajal, funded by the Spanish Ministerio de Ciencia y Tecnología and the University of Alicante, with partial support from the Generalitat Valenciana and the European Regional Development Fund (ERDF/FEDER). This research is partially supported by the Spanish MCyT under grant AYA2002-00814. This publication makes use of data products from the Two Micron All Sky Survey, which is a joint project of the University of Massachusetts and the Infrared Processing and Analysis Center/California Institute of Technology, funded by the National Aeronautics and Space Administration and the National Science Foundation. This research has made use of the SIMBAD database and VizieR Service operated at CDS, Strasbourg, France, and of NASA's Astrophysics Data System Bibliographic Services.

\section{References}

Balona, L. A. 1992, MNRAS, 254, 403

Cox, N. L. J., Kaper, L., Foing, B. H., \& Ehrenfreund, P. 2005, A\&A, 438, 187 Cutri, R. M., Skrutskie, M. F., van Dyk, S., et al. 2003, 2MASS All-Sky Catalog of Point Sources, University of Massachusetts and Infrared Processing and Analysis Center (IPAC/California Institute of Technology), Vizier online catalog II $/ 246$

Dickey, J. M., \& Lockman, F. J. 1990, ARA\&A, 28, 215

Drilling, J. S., \& Landolt, A. U. 1999, in Allen's Astrophysical Quantities, ed. A. N. Cox (Springer), 381

Filliatre, P., \& Chaty, S. 2004, ApJ, 616, 469

Gallo, E., Fender, R. P., \& Pooley, G. G. 2003, MNRAS, 344, 60

Grebenev, S. A., Lutovinov, A. A., \& Sunyaev, R. A. 2003, ATel, 192

Grebenev, S. A., Rodriguez, J., Westergaard, N. J., Sunyaev, R. A., \& Oosterbrock, T. 2004, ATel, 252

González-Riestra, R., Oosterbroek, T., Kuulkers, E., Orr, A., \& Parmar, A. N. 2004, A\&A, 420, 589

Herbig, G. H. 1993, ApJ, 407, 142

in't Zand, J. J. M. 2005, A\&A, 441, L1

Kuulkers, E. 2005, AIP Conf. Proc., 797, 402

Martins, F., Schaerer, D., \& Hillier, D. J. 2005, A\&A, 436, 1049

Masetti, N., Pretorius, M. L., Palazzi, E., et al. 2006, A\&A, 449, 1139

Mathis, J. S. 1999, in Allen's Astrophysical Quantities, ed. A. N. Cox (Springer), 523

Monet, D. G., Levine, S. E., Canzian, B., et al. 2003, AJ, 125, 984

Negueruela, I. 2004, in The Many Scales of the Universe - JENAM 2004 Astrophysics Reviews, ed. J. C. del Toro Iniesta, et al., Proc of the Joint European and Spanish Astronomical Meeting, Granada, Spain, September 2004 [arXiv: astro-ph/0411759]

Negueruela, I., Smith, D. M., \& Chaty, S. 2005, ATel, 429

Negueruela, I., Smith, D. M., Harrison, Th. E., \& Torrejón, J. M. 2006, ApJ, 638, 982

Pandey, M., Manchanda, R., Rao, A. P., Durouchoux, P., \& Ishwara-Chandra, C. H. 2006, A\&A, 446, 471

Petrovic, J., Langer, N., \& van der Hutch, K. A. 2005, A\&A, 435, 1013

Rodriguez, J. 2003, ATel, 194

Schmidt-Kaler, Th. 1982, in Landolt-Börnstein New Series, Group VI, vol. 2b, ed. K. Schaifers, \& H. H. Voigt (Springer-Verlag), 1

Sidoli, L., Paizis, A., \& Mereghetti, S. 2006, A\&A, 450, L9

Smith, D. M. 2004, ATel, 338

Smith, D. M., Heindl, W. A., Markwardt, C. B., et al. 2006, ApJ, 638, 974

Sunyaev, R. A., Grebenev, S. A., Lutovinov, A. A., et al. 2003, ATel, 190

Tody, D. 1993, in Astronomical Data Analysis Software and Systems II, ed. R. J. Hanisch, R. J. V. Brissenden, \& J. Barnes, ASP Conf. Ser., 52, 173

Tokunaga, A. T. 1999, in Allen's Astrophysical Quantities, ed. A. N. Cox (Springer), 143

Vallée, J. P. 2005, AJ, 130, 569

Walborn, N. R., \& Fitzpatrick, E. L. 1990, PASP, 102, 379

Wijnands, R. 2003, ATel, 191

Windhorst, R. A., Burstein, D., Mathis, D. F., et al. 1991, ApJ, 380, 362 